\title{
Kernos
}

Revue internationale et pluridisciplinaire de religion grecque antique

3 | 1990

Varia

\section{Déjouer l'oracle ou la précaution inutile}

\section{Alain Moreau}

URL : http://journals.openedition.org/kernos/996

DOI : 10.4000/kernos.996

ISSN : 2034-7871

\section{Éditeur}

Centre international d'étude de la religion grecque antique

Édition imprimée

Date de publication : 1 janvier 1990

ISSN : 0776-3824

\section{Référence électronique}

Alain Moreau, « Déjouer l'oracle ou la précaution inutile », Kernos [En ligne], 3 | 1990, mis en ligne le 19 avril 2011, consulté le 21 avril 2019. URL : http://journals.openedition.org/kernos/996 ; DOI : 10.4000/ kernos.996 


\section{DÉJOUER L'ORACLE OU LA PRÉCAUTION INUTHE *}

Dans les Choéphores, Oreste fait allusion à «'oracle tout-puissant de Loxias» ${ }^{1}$. Dans la IXe Pythique, plus longuement, Chiron s'adresse ainsi au dieu de Delphes :

toi, qui ne saurais mentir ni errer, ... toi qui sais le terme fatal de toutes choses et tous les chemins qu'elles prennent; toi qui peux compter les feuilles que la terre fait pousser au printemps, et les grains de sable que, dans la mer ou dans les fleuves, roulent les vagues et les souffles des vents; toi qui vois clairement l'avenir et son origine...

(Pind., Pyth., IX, 42-49)

Et la Pythie, consultée par le messager de Crésus, déclame en hexamètres :

Je sais le nombre des grains de sable et les dimensions de la mer. Je comprends le sourd-muet, $j$ 'entends celui qui ne parle pas.

(Hdt., I, 47)

Apollon est omniscient. L'oracle ne se trompe jamais. Toutes les tentatives des mortels pour échapper à une prophétie désastreuse sont vouées à l'échec. C'est la leçon qui se dégage d'un certain nombre de mythes et récits se déroulant selon une structure identique. J'ai constitué un corpus de douze histoires, sept mythes grecs (EEdipe, Althaeménès, Jason, Pâris, Pélops, Persée, Télèphe), un mythe babylonien (Gilgamos, c'est-à-dire Gilgamesh) rapporté par un auteur grec, Élien, quatre récits qui se présentent comme historiques, mais qui sont fortement imprégnés d'éléments mythiques (Agathocle, Cypsélos, Cyrus, Pisistrate).

* Par un heureux hasard (à moins qu'il ne s'agisse d'une continuité dans les centres d'intérêt), les deux ouvrages qui m'ont été les plus utiles ont tous les deux été composés à Liège où se déroule ce Colloque sur "Oracles et mantique» : il s'agit de Marie Delcourt, Edipe ou la légende du conquérant, 2e éd., Paris, Les Belles Lettres, 1981 (1re éd., Bibl. de la Fac. de Philosophie et Lettres de l'Univ. de Liège, Fasc. CIV, Liège, 1944), passim; Roland CRAHAY, La littérature oraculaire chez Hérodote, Paris, Les Belles Lettres, 1956 (Bibl. de la Fac. de Philosophie et Lettres de l'Univ. de Liège, Fasc. CXXXVIII), p. 47, 210, 236-238, 252, 289.

1 EsCH., Ch., 269-270. 


\section{OEdipe}

Le mythe qu'on pourrait qualifier de paradigmatique, et qui est en tout cas le plus illustre, est celui d'CEdipe. L'oracle obtenu par Laïos, avec des variantes, a été cent fois relaté d'Eschyle à Malalas, sur plus d'un millénaire, pour ne pas citer les écrivains contemporains, de Gide à Robbe-Grillet. Nous partirons de la version d'Eschyle, la plus anciennement attestée, mais qui s'inspire sans doute de versions archaïques disparues :

Je pense à la faute ancienne, vite châtiée, et qui pourtant dure encore à la troisième génération, la faute de Laïos, rebelle à Apollon, qui, par trois fois, à Pythô, son sanctuaire prophétique, centre du monde, lui avait déclaré qu'il devait mourir sans enfant, s'il voulait le salut de Thèbes.

(Esch., Sept, 742-749)

On est contraint de compléter cet énoncé, car la formulation de l'oracle eschyléen est elliptique, alors que les vers suivants du stasimon prouvent que la transgression a eu pour résultat, outre la perte de Thèbes, le parricide et l'inceste :

Mais Laïos succombe à un doux égarement, et il engendre sa propre mort, Edipe le parricide, qui a osé ensemencer le sillon sacré où il s'était formé et planter une souche sanglante : un délire unissait les époux en folie!

(Esch., Sept, 750-757)

Or ces conséquences sont annoncées chez Sophocle en ce qui concerne le parricide :

Un oracle arriva jadis à Laïos, non d'Apollon lui-même, mais de ses serviteurs. Le sort qu'il avait à attendre était de périr sous le bras d'un fils qui naîtrait de lui et de moi.

$$
\text { (Soph., O.R., 711-714) }{ }^{2}
$$

Et chez Nicolas de Damas en ce qui concerne l'inceste :

2 Voir aussi : O.R., 1176 : «Un jour, prétendait-on, il tuerait ses parents» (le meurtre de Jocaste étant indirect, puisqu'il s'agit d'un suicide). EuR., Phéniciennes, 17-20: «Le dieu lui répondit : «Ô roi de Thèbes aux beaux chevaux, garde-toi d'ensemencer malgré les dieux le sillon générateur : si tu procrées un fils, cet enfant te tuera et ta maison entière s'abîmera dans le sang»". Voir encore 1595-1599. Pour d'autres allusions à l'oracle, voir C. ROBERT, Oidipus, I, Berlin, 1915, p. 67-68. 
Le dieu dit à Laïos qu'il engendrerait un fils qui le tuerait et qui épouserait sa propre mère.

(90 F 8 Jacoby = Fr. 15 Müller, III, 366)

ainsi que chez Malalas $(2,59)$. Ces deux fragments sont tardifs. Mais on ne doit pas en déduire que l'annonce de l'inceste n'existait pas dans une forme plus ancienne de la légende. On sait en effet que le thème de l'inceste est attesté dès Homère :

Et la mère d'Edipe ! cette belle Épicaste qui, d'un cœur ignorant, commit le grand forfait : elle épousa son fils ! meurtrier de son père, et mari de sa mère ! aussitôt les Immortels révélèrent son crime.

(Od., XI, 271-274) $)^{3}$

L'union avec la mère est annoncée chez Sophocle, sinon à Laïos, du moins à CEdipe :

J'entrerais au lit de ma mère.

(Soph., O.R., 791)

Enfin Marie Delcourt a montré de façon indiscutable que le thème de l'union avec la mère était étroitement associé à celui du héros conquérant dont Edipe constitue l'archétype ${ }^{4}$. On ne peut donc écarter l'hypothèse selon laquelle, dans la version dont s'inspire Eschyle, Laïos était menacé de trois malheurs s'il s'avisait d'engendrer un enfant : sa propre mort sous les coups du fils, le mariage de ce fils avec sa mère, la ruine de sa cité, Thèbes.

On connaît la suite. Malgré ses précautions, Laïos finit par commettre la transgression (dans les mythes et les contes les interdits sont faits pour être transgressés). (Edipe naît. Laïos prend peur. Le nouveau-né est exposé sur le Cithéron ou mis dans un coffre et jeté à la mer ${ }^{5}$, donc voué à une mort quasi certaine. Pourtant l'enfant est sauvé. Il grandit, devient le fils adoptif du roi de Sicyône ou de Corinthe. Dans le récit de Sophocle, alerté par l'insulte malencontreuse d'un compagnon d'ivresse (celui-ci l'a appelé "enfant supposé»), il s'en va consulter l'oracle de Delphes qui lui prédit les deux crimes qui

3 Sur la traduction de ö́p par «aussitôt», voir A. MOREAU, Eschyle : la violence et le Chaos, Paris, Les Belles Lettres, 1985, p. 122-123, n. 86.

4 Voir M. DELCOURT, op. cit., chapitre VI.

5 La version du coffre jeté à la mer, plus rare mais qui se trouve dans d'autres mythes (Danaé et Persée, Augé et Télèphe, Rémus et Romulus, Moïse...) est donnée par HyGN, Fab., 66, qui s'inspire peut-être de l'GEdipe d'EuRIPIDE; schol. EUR., Phen., 26; un bol à reliefs découvert à Tanagra et conservé au Louvre (inv. MNC 660). 
l'attendent, parricide et inceste. Par une aberration dans laquelle il faut voir la marque du destin, il croit se prémunir contre l'oracle en s'éloignant de Corinthe et en se dirigeant vers Thèbes. Dès lors tout s'enchaîne : le meurtre de Laïos, l'inceste avec Jocaste, la naissance des enfants incestueux, la découverte des deux crimes involontaires mais abominables, l'aveuglement, la malédiction lancée contre les fils Étéocle et Polynice, le fratricide et, dix ans après la première expédition argienne contre Thèbes, la prise et la destruction de la cité. L'oracle est accompli. Série de catastrophes qui, à mon avis, sont déjà réalisées ou inscrites en filigrane dans la trilogie eschyléenne. Laïos est assassiné. L'inceste est consommé. Les deux frères se sont entre-tués. Certes Thèbes est sauvée, mais les habitants continuent à avoir peur :

Et une inquiétude étreint la ville : les oracles ne s'émoussent pas.

(Sept, 843-844)

L'oracle d'Apollon avait annoncé la perte de Thèbes si Laïos engendrait un enfant. L'enfant a été engendré : Thèbes périra. La mort de Polynice et d'Étéocle n'y saurait rien changer. Et la présence du mot epigonois au vers 903, quelle qu'en soit la signification dans le contexte, fait évidemment penser à l'expédition des Épigones conduites par Thersandros, le fils de Polynice. La joie de la victoire n'est pas seulement détruite par l'annonce du fratricide, mais encore par la persistance de l'angoisse concernant le sort réservé à la ville et à ses habitants ${ }^{6}$. Eschyle sur ce point reste fidèle à la version épique ${ }^{7}$.

L'analyse narrative permet de dégager une structure :

1. Un individu consulte l'oracle : celui-ci lui prédit la naissance d'un être de sa lignée qui causera le malheur du consultant et des siens.

2. Malgré les précautions prises l'enfant naît.

3. Une tentative est faite pour réparer l'erreur ou l'échec.

4. Cette seconde tentative échoue à son tour.

5. L'oracle s'accomplit : le consultant est tué.

6 Voir A. Moreau, Fonction du personnage d'Amphiaraos dans les Sept contre Thèbes : le "blason en abyme», in BAGB (1979), 2, p. 158, n. 1; Eschyle, p. 225; CR de B. DEForge, Eschyle, poète cosmique, Paris, 1986, in Transe et Théâtre, GITA, $\mathrm{n}^{\circ} 4,1988$, p. 227.

7 La victoire des Épigones est attestée dès HoMÈre. Diomède, fils de Capanée, déclare : «Nous nous flattons, nous, de valoir bien mieux que nos pères. C'est nous qui avons pris Thèbes, la ville aux sept portes» (HOM., Il., IV, 405-406). 
6. Le meurtrier est lui-même destiné au malheur. La race est maudite.

Dans le mythe d'Edipe les précautions prises par Laïos sont doublées de celles que croit prendre Edipe quand, après être allé demander conseil à Apollon, il fuit loin de la cité où il a été élevé.

\section{Althaeménès}

Cette structure, nous la retrouverons dans l'histoire d'Althaeménès, telle qu'elle est racontée par le pseudo-Apollodore et Diodore de Sicile ${ }^{8}$. Selon Apollodore, Katreus, fils de Minos et roi de Crète, avait trois filles, Aeropé, Clyméné, et Apemosyné, et un fils, Althaeménès. L'oracle lui apprend qu'il mourra de la main de l'un de ses enfants. Katreus cache cette prédiction, mais Althaeménès l'apprend et, craignant de devenir le meurtrier de son père, quitte la Crète avec sa sœur Apemosyné et s'installe à Rhodes. Sa sœur est violée par le dieu Hermès; Althaeménès refuse de croire à ses explications et la tue. Devenu vieux, Katreus veut transmettre sa souveraineté. Il débarque à Rhodes mais les bergers le prennent pour un pirate et lancent des traits contre lui. Althaeménès survient et tue son propre père de son javelot. Par la suite, ayant appris la vérité, il est englouti sur sa prière dans un gouffre par les dieux. Dans la version de Diodore, ne pouvant supporter la grandeur de son malheur, il rompt toute relation avec les hommes, et erre seul dans les lieux déserts jusqu'à ce qu'il meure de chagrin. Plus tard les Rhodiens lui accordent les honneurs dus aux héros.

De l'oracle au parricide, le schéma est semblable à celui du mythe d'EEdipe. Dans la version de Diodore la ressemblance est encore plus frappante, puisque l'attitude d'Apollon à l'égard d'Althaeménès est la même qu'à l'égard d'Edipe : il ne répond pas à la question posée, mais lui annonce qu'il tuera son père. Et l'errance d'Althaeménès après la découverte du parricide fait penser à celle d'CEdipe après la révélation de ses deux crimes.

\section{Jason}

Pélias a reçu de Delphes l'avis d'avoir à se défier de l'homme qui paraîtrait devant lui chaussé d'une seule sandale 9 . Or son neveu Jason

8 APOLLOD., Bibl., III, 2, 1-2; DIOD. SIC, V, 59, 1-4.

9 Pind., Pyth., IV, 71-78; PhéRécyde, 3 F 105 Jacoby; APOLL. RH., I, 5-7; HyGin, Fab., 12; LACTANCE Placide, schol. à STACE, Theb., III, 516; V, 336; SERVIUS à VIRG., Buc., IV, 34; second mythographe du Vatican, 135; Tzétzès à LYCOPHRON, 175; ZÉNOBIUS, Cent., IV, 92. 
se rend un jour à Iôlkos, capitale du royaume de Pélias, soit pour revendiquer la souveraineté injustement enlevée autrefois à son père Aeson par Pélias dans la version de Pindare, soit pour prendre part au festin que le roi offre à son père Poséidon dans la version d'Apollonios de Rhodes ${ }^{10}$. Pélias reconnaît l'homme dangereux annoncé par l'oracle et l'envoie en Colchide pour ramener la toison d'or : mission impossible où normalement Jason aurait dû trouver la mort. Mais Jason revient triomphant et la barbare Médée, qu'il a ramenée avec lui, le venge en provoquant la mort de Pélias, tué, découpé et bouilli par ses propres filles. Jason n'est pas, comme EEdipe ou Althaeménès, écrasé de douleur devant ce crime qu'il a voulu ou laissé faire, mais on ne peut pas dire que la suite de sa vie soit très heureuse. Si dans la forme la plus anciennement attestée du mythe, celle d'Hésiode ${ }^{11}$, il coule des jours heureux à Iôlkos, dans les versions classiques et post-classiques, il voit ses enfants massacrés par sa propre épouse, périt dans l'incendie du palais royal de Corinthe ou bien est écrasé par les débris d'Argô, ou encore se suicide.

\section{Pâris}

Hécube, épouse de Priam, le roi de Troie, rêve qu'elle accouche d'une torche ${ }^{12}$. Le feu s'étend à toute la cité et la brûle entièrement. Priam se fait expliquer le songe par des devins ${ }^{13}$. On lui annonce que l'enfant qui naîtra provoquera la ruine de sa patrie et qu'il faudra l'exposer ${ }^{14}$. À

10 PIND., Pyth., IV, 101 sq.; APOLL. RH., I, 12-14.

11 Hés., Théog., 992-1002. Voir A. MoREAu, Les mille et une facettes de Médée, in ConnHell, 27 (1986), p. 78.

12 Sur le songe d'Hécube et l'exposition de Pâris les principales sources sont APollod., Bibl., III, 12, 5; PIND., Péans, 8, éd. Puech, p. 128 (PINDARE y parle non d'une torche, mais d'une Érinys incendiaire, aux cent bras); schol. A HOM., Il., III, 325 = ASCLÉPIADE DE TRAGILOS, 12 F 12 Jacoby; LYCOPHRON, Alex., 13621363; TZÉTZÈs, schol. à LYCOPHRON, 86, 224, 913; EUR., Andr., 293-300 (la prophétie y est attribuée à Cassandre, ce qui est invraisemblable si l'on rassemble toutes les données du mythe : la jeune fille violée par Ajax, fils d'Oilée, et aimée d'Agamemnon aurait une quarantaine d'années...); schol. M,A, EUR., Andr., 293; Troyennes, 919-923; Iph. Aul., 1284-1288. EURIPIDE avait consacré une tragédie entière à cette première partie de la vie de Pâris jusqu'au retour à Troie, l'Alexandros (voir F. JouAn, Euripide et les Chants Cypriens, Paris, 1966, p. 113-142); ENNIUS, Alexander, Fr. 38-48; VIRG., En., VII, 319-22; OVIDE, Hér., XVI, 237-40; HYGIN, Fab., 91, 249; SÉNĖQUE, Agam., 706; SERVIUS, à VIRG., En., VII, 320; X, 705; second mythographe du Vatican, 197.

14 L'ordre vient d'Apollon dans la version d'ENNIUS. 
la naissance de l'enfant, Priam obéit : Pâris est exposé sur le Mont Ida. Mais nourri par une ourse, et recueilli ensuite par le vieux serviteur qui l'avait exposé ou par un bouvier, Pâris grandit, gardant des troupeaux sur le Mont Gargaros, le plus haut sommet de l'Ida. Plus tard, il regagnera Troie et, par le rapt d'Hélène, sera la cause de la destruction de sa cité et de la mort de Priam, tué par Pyrrhos/Néoptolème. À ce moment-là, Pâris est déjà mort, atteint par la flèche empoisonnée de Philoctète ${ }^{15}$.

\section{Pélops}

Un oracle avait prédit à Oinomaos, roi de Pise et père d'Hippodamie, qu'il serait tué par son futur gendre ${ }^{16}$. C'est pourquoi il impose aux prétendants une course de char. Le prétendant obtiendra la main d'Hippodamie si, monté sur son char en compagnie de la jeune fille, il réussit à atteindre l'isthme de Corinthe avant Oinomaos. Mais celui-ci, qui possède des chevaux merveilleux donnés par Arès, rejoint toujours le prétendant avant le but, malgré le handicap de départ, le tue et expose sa tête sur la façade de son palais. Mais un jour arrive le beau Pélops, dont Hippodamie tombe amoureuse. Elle persuade Myrtilos, cocher d'Oinomaos, de lui apporter son aide et le roi trouve la mort, traîné par les rênes du char saboté. Avant de mourir, il a le temps de maudire Pélops et sa race. Malédiction pleinement exaucée, comme le prouve la simple énumération des descendants de Pélops : Atrée, Thyeste, Agamemnon, Ménélas, Égisthe, Oreste...

\section{Persée ${ }^{17}$}

Acrisios, roi d'Argos, apprend par l'oracle que l'enfant qui naîtra de sa fille Danaé causera sa mort. Il enferme celle-ci dans une chambre souterraine en bronze. Mais la jeune fille est séduite par Zeus qui s'unit

15 Le combat de Philoctète et de Pâris était déjà rapporté dans la Petite Iliade de LESCHìs, voir le résumé de PRoclos (Homeri opera, V, éd. Allen, p. 106).

16 C'est la version de Diodore (IV, 73). Dans d'autres versions c'est parce qu'il aime sa fille d'amour qu'il ne veut pas lui donner d'époux, voir infra, p. 277. Sur ce mythe, voir A. MOREAU, Épouser la princesse : Pélops et Hippodamie, in Femmes et patrimoine dans les sociétés rurales de l'Europe Méditerranéenne, Paris, CNRS, 1987, p. 227-287.

17 Sur cette partie du mythe de Persée, voir A. MOREAU, Le discobole meurtrier, in Pallas, 34 (1988), p. 2, avec la note 12; R. AÉLION, Quelques grands mythes héroïques dans l'œuvre d'Euripide, Paris, Les Belles Lettres, 1986, p. 151-83 (avec bibliographie, p. 183); J.-J. MAFFRE, Akrisios, in LIMC, I, 1, p. 449-52. 
à elle sous la forme d'une pluie d'or ${ }^{18}$. Les unions avec les dieux étant toujours fécondes, un enfant naît, Persée. Acrisios l'apprend et fait exposer la mère et l'enfant dans un coffre qu'il lance sur les flots. Le coffre aborde dans l'île de Sériphos. Persée grandit et, au terme de multiples exploits dont le plus célèbre est la décapitation de Méduse, il veut revoir son aieul. En dépit des bonnes intentions du petit-fils, Acrisios, qui craint toujours l'oracle, s'enfuit. Mais un jour leurs destins se rejoignent. Acrisios assiste à des jeux auxquels participe Persée, en tant que discobole. Le vent fait dévier le disque. Acrisios, frappé à la tête ou au pied meurt. D'après Apollodore, Persée eut honte de réclamer l'héritage de celui qui était mort de sa main, et échangea le royaume d'Argos contre celui de Tirynthe, qui appartenait à Mégapenthès, fils de Proitos. Il ne semble pas que Persée ait connu les malheurs qu'ont eu à subir les héros des histoires précédentes. C'est seulement dans la version tardive de Jean Malalas qu'il est vaincu et tué par Képheus, le père d'Andromède ${ }^{19}$. Mais si le mythe diffère sur ce point des autres, il se révèle aussi paradigmatique que le mythe d'Edipe en ce qui concerne le thème de la précaution inutile. Il présente en effet une triplication du thème : l'enfermement de Danaé, l'exposition sur les flots, la fuite du roi, et, au bout du compte, comme toujours, la mort.

\section{Télèphe}

L'histoire de Télèphe ressemble à celle de Persée, avec cette différence toutefois que l'oracle n'y apparaît que dans une seule source, le rhéteur Alcidamas ${ }^{20}$. D'après Alcidamas l'oracle de Delphes prédit à Aléos, roi de Tégée, que sa fille, Augé, mettra au monde un enfant qui

18 Principale sources : SIMONIDE, fr. 38 Page (= 543); PHÉRÉcYde, 3 F 10-12 Jacoby = schol. APOLl. RH., IV, 1091, 1515; APOLloD., II, 4, 1-5; PAUS., II, 16, 2; ZÉnoBIUs, Cent., I, 41 (éd. Leutsch-Schneidewin, Paroemiographi Graeci, I, 1517); Hygin, Fab., 63, 273; LaCtance Placide à STACe, Théb. , I, 255, éd. Jahnke. SOPHOCLE avait composé un Acrisios et une Danaé, EURIPIDE une Danaé. Il en reste quelques fragments. Sur l'existence d'une Danaé dans la tétralogie consacrée par EschYle à Persée, voir R. AÉlion, Euripide héritier d'Eschyle, Paris, Les Belles Lettres, 1983, I, p. 266-69; B. Deforge, op. cit., p. 134-135. Il nous reste des fragments des Tireurs au filet, drame satyrique racontant l'arrivée de Danaé et Persée à Sériphos. Selon certains, dit APollodore (II, 4, 1), le séducteur serait Proitos. Voir aussi schol., Il., XIV, 319 = PIND., fr. 284 Snell. Mais la majorité des sources rapportent l'épisode de Zeus métamorphosé en pluie d'or.

19 J. Malalas, 2, 38-39 (J.-P. Migne, Patrol. Gr., 97, col. 109).

20 Alcidamas, Discours d'Ulysse contre Palamède, 4 (éd. Radermacher, Artium Scriptores, Vienne, 1951, p. 141 sq.). 
tuera ses oncles, les fils d'Aléos. Pour empêcher ce malheur, le roi fait d'Augé la prêtresse d'Athéna et la menace de mort si elle perd sa chasteté. C'est alors que survient Héraclès qui se rendait à Élis (pour faire la guerre à Augias). Il est reçu par Aléos dans le temple d'Athéna et, pris d'ivresse, viole la prêtresse. Apprenant que sa fille est enceinte, Aléos envoie chercher Nauplios (frère de Palamède) et donne à celui-ci l'ordre de jeter Augé dans les flots. Nauplios emmène la jeune femme mais, en route, Augé donne naissance à Télèphe sur le Mont Parthénios. $\mathrm{Au}$ lieu de tuer la mère et le fils, Nauplios les vend à Teuthras, roi de Mysie, qui, étant sans enfant, épouse Augé et adopte Télèphe. Les autres versions ignorent l'oracle et commencent le récit à partir du viol d'Augé par Héraclès ${ }^{21}$. Après sa naissance, l'enfant est exposé sur le Mont Parthénios, nourri par une biche, puis recueilli par des bergers. Ou bien la mère et l'enfant sont mis dans un coffre abandonné aux flots. Le coffre est emporté jusqu'à la côte mysienne, dans le pays de Teuthras. Télèphe grandit et plus tard tue ses oncles maternels, comme l'avait prédit l'oracle dans la version d'Alcidamas. Nous n'avons pas conservé le récit détaillé de ce meurtre, mais il est mentionné par Hygin, un auteur de proverbes ${ }^{22}$, et on a tout lieu de croire qu'il faisait l'objet d'une tragédie perdue de Sophocle, les Aléades. On peut reconstituer sommairement les faits de la façon suivante : Tégée, parvenu à l'âge adulte, se rend chez son oncle Aléos à Tégée. Là, il tue accidentellement ses oncles maternels ${ }^{23}$ et retourne en Mysie pour se faire purifier. Un fragment attribué aux Mysiens d'Eschyle ${ }^{24}$ fait allusion au silence de Télèphe sur le trajet de Tégée à la Mysie. Le silence fait très certainement partie d'un rite préparant à la purification ${ }^{25}$.

21 Sur cet épisode mythique très complexe, voir la note de Frazer à l'édition d'APOLLODORE (ח, 7, 4), I, p. 252-255; SCHMIDT, Telephos, in ROSCHER, Lexicon, $\mathrm{V}$, col. 275-277; SCHWENN, in RE, V, A1, col. 362-363.

22 HYGIN, Fab., 244, éd. Leutsch-Schneidewin, Paroemiographi Graeci, I, p. 212.

23 Hippothoos et Nerea (éd. Muncker). Mais Rose pense que le mot «Nerea» est apocryphe. APOLLODORE (III, 9, 1) les appelle Kepheus et Lycurgue. Chez Apollonios de RhOdes (I, $161 \mathrm{sq}$.) et PAUSANias (VIII, 4, 4), il y a trois fils, Kepheus, Lycurgue et Amphidamas.

24 Fr. 415 Mette $=$ Nauck 2 , p. $47=$ Radt, p. 257.

25 Voir FrAZER, op. cit., p. 254-255. 


\section{Gilgamesh}

L'histoire est rapportée par Élien ${ }^{26}$. Sévéchoros était roi de Babylone. Des Chaldéens lui annoncent que le fils de sa fille enlèvera la souveraineté à son grand-père. Pour se prémunir, le roi, effrayé, comme Acrisios (la comparaison est faite par Élien lui-même), emprisonne sa fille et la fait surveiller étroitement. Mais, pas plus que Danaé, l'enfermement n'empêche la princesse d'être fécondée par un homme et d'accoucher secrètement d'un garçon. Les gardiens de la tour, craignant la colère du roi, se débarrassent de l'enfant en le jetant du haut de la citadelle. Mais un aigle, grâce à sa vue perçante, se rend compte de la chute du nourrisson et, avant qu'il ne s'écrase au sol, se glisse sous lui, l'installe sur son dos, l'emmène jusqu'à un jardin et le dépose avec précaution. Le jardinier voit le bel enfant, se prend d'affection pour lui, l'élève et lui donne le nom de Gilgamos. Gilgamos, c'est-à-dire Gilgamesh, devient plus tard le tout-puissant roi de Babylone. Élien ne rapporte pas les conditions dans lesquelles il s'empare du pouvoir.

\section{Agathocle}

Les enfances de ce personnage historique sont racontées par Diodore 27 . Carcinos de Rhégion, exilé de sa patrie, s'est établi à Thermes en Sicile, cité soumise aux Carthaginois. Il a pris pour maîtresse une femme du pays. Or, alors que celle-ci va avoir un enfant, il a toutes les nuits des cauchemars. Il fait interroger l'oracle de Delphes, qui répond que l'enfant sera cause de grands malheurs pour les Carthaginois et toute la Sicile. Pris de peur, Carcinos fait exposer le nouveau-né et place des gardes près de lui pour veiller à ce qu'il meure. Mais au bout de quelques jours, l'enfant étant toujours vivant, les gardes relâchent leur attention et la mère réussit à s'en emparer. L'enfant est élevé chez son oncle maternel qui lui donne le nom d'Agathocle. Agathocle est séduisant de traits et robuste de corps. Quand il a sept ans, sa mère révèle toute la vérité à Carcinos qui, à la vue de l'enfant, regrettait amèrement celui qu'il avait exposé. C'est pourquoi il reprend avec joie son fils mais s'exile à Syracuse par crainte des Carthaginois. Comme il est pauvre, il lui apprend le métier de potier. En 316 av. J.-C., Agathocle devient le tyran de Syracuse pour le malheur de la Sicile :

26 ÉLIEN, De la nature des Animaux, XII, 21.

27 DIOD., XIX, 2. 
Aucun tyran avant lui n'avait, en effet, accompli rien de tel, ni fait montre de tant de cruauté envers ses sujets : il punissait les particuliers en exécutant toute la famille, châtiait les cités en massacrant tous les hommes adultes et, en obligeant, pour quelques accusés, la masse de la population, même parfaitement innocente, à subir les mêmes malheurs, il condamnait à mort les cités tout entières.

(Diod. Sic., XIX, 1, 8)

\section{Cypsélos}

Selon Hérodote ${ }^{28}$, les Bacchiades, oligarchie régnant à Corinthe, avaient reçu de Delphes l'oracle suivant, auquel ils ne comprenaient rien :

Une aigle est grosse au milieu des rochers; elle enfantera un lion fort et féroce qui rompra sous eux les genoux de beaucoup. Pensez-y bien, Corinthiens, qui habitez autour de la belle Pirène et de la sourcilleuse Corinthe.

Peu de temps après, un habitant de Corinthe, qui, comme Laïos, était allé consulter l'oracle de Delphes pour savoir s'il pourrait avoir un enfant, reçut un oracle tout aussi obscur :

Éétion, personne ne t'honore, bien que tu mérites grandement d'être honoré. Labda est grosse; elle enfantera une pierre roulante, qui s'abattra sur les hommes régnants et châtiera Corinthe.

Ayant eu connaissance de ce second oracle, les Bacchiades compren-

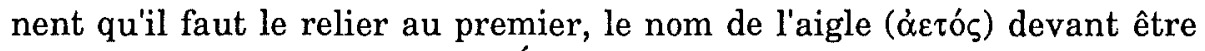
mis en rapport avec celui d'Éétion ('Actí $\omega v$ ), et le nom des rochers ( $\pi \dot{\varepsilon} \tau \rho \circ)$ avec celui du dème de Pétra où habite Éétion. C'est pourquoi ils décident de se débarrasser de l'enfant. À la naissance de celui-ci, ils envoient dix des leurs chez Éétion. Labda, la mère (qui appartenait aussi à la famille des Bacchiades) ignorant la raison de leur venue, leur présente l'enfant, sur leur demande. Mais celui qui avait pris le bébé dans ses bras en a pitié et le remet à son voisin qui, pour les mêmes raisons, le remet à un autre. Quand les dix envoyés se sont récusés, ils rendent l'enfant à sa mère, puis sortent et se disputent, accusant surtout le premier d'avoir failli à sa mission. Labda, qui avait tout entendu, cache son bébé dans un coffre ( $\kappa \cup \psi \varepsilon \dot{\lambda} \lambda \eta)$. Les envoyés, rentrés dans la maison, cherchent partout, sans parvenir à mettre la main sur l'enfant et prennent la résolution de déclarer aux Bacchiades qu'ils ont exécuté

28 HDT., V, 92. 
l'ordre. L'enfant, en souvenir du coffre qui l'avait sauvé, fut nommé Cypsélos. Devenu adulte, il attaqua Corinthe et s'en empara :

Devenu tyran, il bannit beaucoup de Corinthiens, en priva beaucoup de leurs biens, et bien davantage de la vie.

\section{Cyrus}

La source du récit est encore Hérodote ${ }^{29}$. Astyage, roi des Mèdes, rêve que sa fille, Mandane, urine avec tant d'abondance que sa ville en est inondée, et même l'Asie tout entière submergée. Les mages qui interprètent ce songe le déclarent funeste. Aussi, plus tard, Astyage se garde-t-il de marier Mandane à un prince puissant mais la donne à un Perse de bonne condition, certes, mais qu'il juge inférieur à un Mède, Cambyse. Dans l'année qui suit, Astyage fait un second rêve. Il lui semble que du sexe de sa fille pousse un cep de vigne, et que cette vigne s'étend sur toute l'Asie. Les mages annoncent que l'enfant qui naîtra sera roi à sa place. C'est pourquoi, à la naissance de Cyrus, Astyage ordonne à un homme de sa parenté, Harpage, d'emporter le bébé et de le tuer. Le récit continue alors à se dérouler selon la structure déjà maintes fois dégagée. Harpage enjoint à un bouvier d'exposer l'enfant. Sur la prière de sa femme, le bouvier substitue son propre fils, mort-né, à Cyrus. Celui-ci grandit. Plus tard Astyage apprend la vérité, se venge cruellement d'Harpage qui n'a pas rempli sa mission et interroge à nouveau les mages pour savoir quel sort il doit réserver au jeune Cyrus. Les mages considèrent que l'enfant, ayant été roi des enfants du village au cours du jeu qui entraîna la découverte de son identité, l'oracle est accompli. Rassuré, Astyage renvoie Cyrus, âgé de dix ans, en Perse chez ses véritables parents qui, à partir du nom de Kyno, la femme du bouvier, font courir le bruit que Cyrus, exposé, avait été nourri par une chienne $^{30}$. Cyrus, devenu adulte, fomente une révolte des Perses contre les Mèdes, sur les conseils d'Harpage. Astyage, vaincu et fait prisonnier, doit céder son royaume à son petit-fils Cyrus.

29 HDT., I, 107-130.

30 Version rationaliste d'un récit qui est plus mythique qu'historique. L'animal nourricier appartient certainement à la forme ancienne de la légende, comme dans les mythes de Pâris, Télèphe ou Romulus et Rémus. 


\section{Pisistrate}

Un fois de plus le récit se trouve chez Hérodote ${ }^{31}$, mais il présente une différence de taille par rapport au schéma étudié jusqu'à présent. Le départ est le même : une prédiction. Hippocrate d'Athènes, assistant aux fêtes d'Olympie, offre un sacrifice. Et voici que les chaudrons étant dressés, remplis de chair et d'eau, mais sans feu allumé, ils se mettent soudain à bouillir et débordent. Chilon de Lacédémone, l'un des sept sages, qui se trouve là par hasard, donne alors à Hippocrate le conseil de ne pas épouser une femme qui puisse avoir des enfants; s'il en a une, de la renvoyer; et s'il a un fils, de le répudier. Mais, et c'est ici que le récit diverge du schéma habituel, Hippocrate ne suit pas le conseil de Chilon. Par la suite naît un fils, Pisistrate, qui plus tard devient le tyran d'Athènes.

Essayons maintenant de dessiner la structure commune qui se dégage de ce corpus de douze récits :

1. La prédiction : elle est présente partout, sous la forme d'une consultation de l'oracle de Delphes, ou bien de l'interprétation de songes, de prodiges par des devins, mages.

2. À neuf reprises (Eđdipe, Pâris, Persée, Télèphe, Gilgamesh, Agathocle, Cypsélos, Cyrus, Pisistrate), la prédiction annonce la naissance d'un enfant maléfique.

3. Le personnage maléfique est toujours apparenté au consultant, dont il est le fils (Edipe, Althaeménès, Pâris, Agathocle, Pisistrate), le petit-fils (Persée, Télèphe, Gilgamesh, Cyrus), le neveu (Jason), le futur gendre (Pélops), le petit-neveu (Cypsélos : Labda, sa mère, est fille d'Amphion, un Bacchiade).

4. La prédiction est sinistre : perte du pouvoir ou même de la vie pour le consultant (sauf dans les cas d'Agathocle et de Pisistrate), asservissement et/ou ruine de sa cité.

5. Sauf dans le cas de Pisistrate, le consultant tente de se prémunir contre l'oracle. Parfois la précaution est redoublée ou même triplée. Ainsi dans le mythe d'Edipe ou celui de Persée.

6. L'oracle s'accomplit toujours.

7. Dans beaucoup de cas (surtout les mythes grecs), l'être qui est l'objet de la prédiction finit lui-même fort mal.

31 HDT., I, 59. 
La leçon que l'on tire de cet ensemble paraît, à première vue, appartenir à la sagesse des nations : on n'échappe pas à sa destinée. C'est contre cette croyance que se révolte La Fontaine dans l'Horoscope $(F a b ., \text { VIII, } 6)^{32}$, tout en en donnant deux illustrations, celle de l'enfant que, sur la foi d'une prédiction, son père protège contre les lions, lui interdisant la chasse, et qui meurt blessé par un clou caché sous une tapisserie représentant un lion; celle du dramaturge Eschyle qui, pour échapper à une prédiction le menaçant de la chute d'une maison, dort dans les champs, et a le crâne fracassé par une tortue jetée par un aigle. C'est aussi la leçon que développe Hérodote dans l'histoire du fils de Crésus dont une prédiction a annoncé la mort par le fer et qui sera tué par le javelot de celui qui précisément était chargé de le protéger ${ }^{33}$. C'est encore la leçon des contes : les parents de la belle au bois dormant ne peuvent empêcher leur enfant de se faire piquer par le fuseau funeste annoncé par la prédiction de la mauvaise fée. Mais comme il s'agit d'un conte, la mort est remplacée par un sommeil de cent ans.

Le corpus des douze récits est traversé aussi par d'autres thèmes importants. Thème de l'enfant maléfique qui apporte le malheur sur sa race ou sa patrie ${ }^{34}$. Le caractère funeste de l'enfant se révèle parfois par le loimos, stérilité ou peste qui s'abat sur le pays. C'est le point de départ de l'Edipe-Roi de Sophocle et le fléau sévit aussi dans le mythe de Télèphe : d'après Apollodore ${ }^{35}$, l'exposition de Télèphe dans le temple d'Athéna a pour conséquence un loimos qui ravage le pays. Thème de l'épreuve : l'enfant exposé sur la montagne ou sur les flots, s'il échappe à la mort, est promis aux plus hautes destinées : il deviendra roi ou tyran $^{36}$. Le plongeon subi par Gilgamesh est une autre forme d'épreuve qui habilite au pouvoir ou même peut apporter l'immortalité. Il est

32 «e ne crois point que la nature

Se soit liée les mains, et nous les lie encor,

Jusqu'au point de marquer dans les cieux notre sort».

33 Sur l'anecdote concernant Eschyle, voir Vie anonyme d'Eschyle, § 9; Souda, s.v.

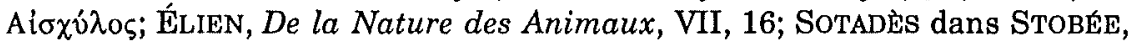
Floril., 98, 9; Pline l'ANCIEN, N.H., X, 3; VAlère MaXime, IX, 12. Sur le fils de Cyrus : HDT., I, 34-45. Voir A. MOREAU, Étymologie et mythe originel : Adraste, Andromaque, Déjanire, in Sens et pouvoirs de la nomination dans les cultures hellénique et romaine, Actes du Colloque de Montpellier, 23-24 mai 1987, Montpellier, 1988, p. 108-113.

34 Voir M. Delcourt, Stérilités mystérieuses et naissances maléfiques dans l'Antiquité Classique, Paris, Les Belles Lettres, 1986 (Bibl. de la Fac. de Phil. et Lettres de l'Univ. de Liège, Fasc. LXXXIII) (1re éd., Liège, 1938).

35 APOLLOD., II, 7, 4; III, 9, 1.

36 Voir M. DelcourT, Edipe..., p. 1 sq. 
présent dans les mythes d'Eumolpos, Glaucos le Marin, Mélicerte, Thésée, Héphaïstos, Dionysos. Il est redoublé dans l'histoire de Gilgamesh, qui plonge au fond de l'eau pour aller cueillir la plante d'immortalité ${ }^{37}$. L'enfant qui survit à ce type d'épreuve est un élu (ce qui ne l'empêche pas d'être aussi, parallèlement, l'enfant maléfique, le réprouvé : similitude des extrêmes). Cette élection divine s'exprime aussi par l'étrange protection animale : l'ourse pour Pâris, la biche pour Télèphe, l'aigle pour Gilgamesh, la chienne pour Cyrus.

Dans ces conditions, on pourrait penser qu'Hérodote et Diodore, lorsqu'ils rapportent des enfances de tyrans calquées sur celles de héros de la mythologie se font les propagandistes involontaires de la tyrannie. En réalité, ils précisent bien que les règnes d'Agathocle et de Cypsélos furent une catastrophe pour leur cité : bannissements, spoliations et massacres s'y multiplièrent. En revanche, comme le pense Roland Crahay ${ }^{38}$, la narration qu'Hérodote fait de la prise du pouvoir par Pisistrate à Athènes a sans aucun doute pour sources, au moins partiellement, des récits véhiculés par la propagande du tyran, ce qui explique les différences avec le schéma habituel : le consultant ne cherche pas à infléchir le destin; le thème de la précaution inutile en effet est absent; Pisistrate prend le pouvoir et se révèle un bon dirigeant :

Pisistrate, dès lors, régna sur les Athéniens, sans troubler l'exercice des magistratures existantes, sans changer les lois; il gouverna la cité en respectant la constitution établie, et l'administra excellemment.

(Hdt., I, 59)

Pourtant Hérodote dit aussi que le peuple athénien est «asservi et divisé»39. Et il est nettement moins élogieux que Thucydide et Aristote $^{40}$. Il ne dit pas, comme eux, que le gouvernement de Pisistrate fut l'âge d'or, le «règne de Cronos». Il ne range pas Pisistrate parmi les sept sages. On peut donc qualifier d'hétéroclite l'information d'Hérodote sur ce personnage.

En fait, l'idée essentielle qui sous-tend le corpus des mythes de la précaution inutile ne me semble être ni le précepte de la sagesse des nations sur la destinée inévitable, ni le thème de l'enfant maléfique, ni

37 Voir A. Moreau, Les mille et une facettes de Médée, in ConnHell, 26 (1986), p. $22-23$.

38 R. CRAHAY, op. cit. n. 1, p. 254.

39 HDT., I, 59.

40 Voir G. GLotz-R. CoHen, Histoire Grecque, I, Des origines aux guerres médiques, 4e éd., Paris, PUF, 1948 (1re éd., 1929), p. 448-449, 461. 
celui de l'épreuve habilitant au pouvoir, ni une propagande politique au service des tyrans ou de leurs adversaires. Il faut prendre garde à une caractéristique du corpus : les deux tiers des récits ont pour point de départ un oracle de Delphes. Or il y a de fortes chances pour que les mythes rassemblés ici soient nés longtemps avant l'installation d'Apollon à Delphes. Et même, dans nos sources les plus anciennes, pourtant postérieures à cette installation, le dieu n'est pas présent. C'est ainsi que la prédiction faite à Laïos par Apollon n'apparaît ni chez Homère, ni dans les fragments de la Thébaïde et de l'GEdipodie, ni chez les lyriques jusqu'à la IIe Olympique de Pindare (476 av. J.-C.). Dans la scholie des Phéniciennes, 1760, attribuée à Pisandre de Laranda, il n'est pas question de l'oracle d'Apollon, mais de la faute commise par Laïos à l'égard d'Héra Gamostolos quand il enleva Chrysippe et d'une prédiction de Tirésias. Il n'est pas exclu que la première forme du mythe ait été non une prophétie mais la malédiction proférée par Pélops après le suicide de Laïos, malédiction qu'on trouve rapportée dans une hypothésis des Sept et une hypothésis des Phéniciennes, tandis qu'une autre hypothésis des Phéniciennes et une scholie du Laurentianus à la fin d'Edipe-Roi contaminent les deux versions : l'oracle d'Apollon y annonce que Zeus a ratifié les malédictions de Pélops. D'après Lucien Legras, la Thébaïde, épopée du cycle, donnerait pour cause aux malheurs de Laïos les malédictions de Pélops, tandis que l'Edipodie, poème plus tardif, composé à l'époque où la renommée de l'oracle de Pythô se répandait partout, attribuait ses malheurs à la violation de l'ordre d'Apollon ${ }^{41}$.

Les mêmes remarques sur l'absence de l'oracle de Delphes à époque ancienne peuvent être faites à propos des autres mythes. Dans le mythe de Jason, si le mythème de l'homme au pied nu me semble ancien (car il suggère une relation avec le monde chthonien dans un contexte initiatique $)^{42}$, cela ne veut pas dire qu'il ait été lié originellement à l'oracle de Delphes. Celui-ci apparaît seulement au début du Ve siècle, dans la IVe Pythique de Pindare (vers 71-78) et chez Phérécyde (3 F 105 Jacoby). Dans une version ancienne du mythe Jason n'est même pas responsable de la mort de Pélias : on comprendrait mal que le meurtrier participe aux jeux funèbres en l'honneur de sa victime comme il est

41 Voir L. LEGRAS, Les légendes thébaines dans l'épopée et la tragédie grecques, Paris, 1905, p. 46; voir aussi R. AÉLION, Quelques grands mythes... (voir n. 17), p. 29-34.

42 Voir A. MOREAU, art. cit. n. 37, p. 75. 
attesté sur le coffre de Cypsélos ${ }^{43}$. La version qui explique le comportement d'Oinomaos par un oracle prédisant qu'il serait tué par son futur gendre est en concurrence avec une autre version expliquant ce comportement par l'amour incestueux que le père éprouve pour sa fille ${ }^{44}$. Nous n'avons aucun moyen de savoir si la version qui contient l'oracle a précédé l'autre, puisque la source la plus ancienne mentionnant l'oracle, Phérécyde, est bien trop récente pour nous ramener aux origines du mythe. La même remarque peut être formulée au sujet de Persée : Phérécyde est le premier à faire allusion à l'oracle ${ }^{45}$. Dans le mythe de Télèphe, l'oracle, comme je l'ai dit, apparaît seulement chez Alcidamas, un rhéteur du IV siècle av. J.-C. Quant aux histoires de tyrans, elles sont rapportées par Hérodote, contemporain de Sophocle et de Périclès, ou par Diodore qui, lui, est contemporain de César et d'Auguste.

Quelle conclusion tirer de cette apparition tardive d'Apollon dans le corpus ? Il n'est pas possible d'avoir des certitudes, étant donné le caractère extrêmement lacunaire de nos sources, étant donné aussi que la documentation iconographique, souvent plus ancienne que les sources écrites, ne peut guère nous aider : la consultation de l'oracle n'est ni spectaculaire, ni spécifique, et les artistes n'avaient guère de raison de choisir cet épisode. C'est ainsi que Jean-Jacques Maffre, recensant les représentations d'Acrisios ${ }^{46}$, distingue :

A. Acrisios assistant à la fabrication du coffre.

B. Acrisios devant Danaé et le jeune Persée déjà dans le coffre.

C. Acrisios à côté du coffre.

D. Acrisios seul, au tombeau de Persée ${ }^{47}$.

E. Acrisios parmi les rois d'Argos.

Il n'y a pas de représentation d'Acrisios consultant l'oracle de Delphes.

Les données étant fragiles, nous en sommes donc réduits à des hypothèses. J'émettrais pourtant volontiers celle-ci : la fréquence de l'oracle de Delphes dans les mythes de la précaution inutile et l'absence de cet oracle dans les sources les plus anciennes de ces mythes me paraissent prouver qu'à une époque qui se situe vraisemblablement au

43 Paus., V, 17, 9-11. Voir F. VIAN, éd. des Argonautiques d'Apollonios de Rhodes, Paris, Les Belles Lettres, 1974, Tome I, p. XXXIII; A. MOREAU, art. cit., p. 78-79.

44 C'est ainsi que les deux versions sont rapportées par Apollodore, Epitome, II, 4.

45 PHÉRÉCYDE, 3 F 10 Jacoby.

46 Voir note 17.

47 Probablement un cénotaphe élevé au-dessus de la chambre souterraine. 
Vle siècle et au Ve siècle, au moment où le prestige de l'oracle est à son comble, il y a eu une volonté de mettre ces mythes au service d'Apollon, exactement comme les mythes de l'oracle mal interprété (Hyllos, Aristomachos, Crésus) révèlent la subtilité du dieu et la sottise des hommes ${ }^{48}$. L'oracle ne se trompe jamais, et quand il y a une erreur de prédiction, comme dans l'une des consultations concernant Cyrus, c'est précisément parce qu'il ne s'agit pas de l'oracle d'Apollon, mais de mages orientaux. On peut, comme Jean Defradas, voir là une intervention de la propagande delphique, d'un clergé désireux de maintenir et d'augmenter la prospérité de Delphes ${ }^{49}$. On peut aussi, comme Georges Roux, mettre en avant l'action désintéressée de "Delphiens de ccur et d'esprit», les grands poètes, historiens, philosophes de la Grèce, Pindare, Eschyle, Hérodote, voire, plus tardivement, Platon ${ }^{50}$. Pour les Grecs de cette époque Apollon est le dieu qui dit la vérité. Toute tentative pour échapper à ses prophéties est vouée à l'échec et même précipite la perte de l'auteur de la tentative : c'est en fuyant Persée qu'Acrisios rencontre la mort.

Fatalisme, diront certains. Mais les leçons que dégagent du mythe paradigmatique d'EEdipe les grands écrivains du Ve siècle me conduisent à écarter cette notion pour la période classique. Certes Pindare rappelle le temps où "le fils prédestiné ( $\mu$ ópıнos) de Laïos rencontra son père et le tua, pour accomplir l'antique oracle proféré à Pythô» 51 , mais le passage se situe dans un contexte où il est question des vicissitudes de la vie, de l'impossibilité de prévoir si le bonheur présent sera durable. On n'est pas très loin de la sentence finale d'Edipe-Roi :

Gardons-nous d'appeler jamais un homme heureux, avant qu'il ait franchi le terme de sa vie sans avoir subi un chagrin52.

48 Hyllos ne comprend pas que «la troisième moisson» signifie «la troisième génération" (APOLLOD., II, 8, 2); Aristomachos ne comprend pas que l'attaque par «la voie étroite» signifie «par les détroits», et non «par l'isthme» (APOLLOD., II, 8, 2); Eusèbe DE CÉSARÉE, Préparation évangélique, V, 20; Crésus ne comprend pas que le grand empire qu'il détruira est le sien et que le mulet roi des Mèdes est Cyrus, fils d'un Perse et d'une Mède (HDT., I, 53-56, 91). L'erreur d'interprétation d'Hippias (le rêve d'union avec la mère ne signifie pas reconquête de la patrie, mais plus prosaïquement une dent perdue dans le sable, HDT., I, 107) n'est pas mise en rapport avec un oracle d'Apollon.

49 J. DeFradas, Les thèmes de la propagande delphique, Paris, 1954.

50 G. Roux, Delphes, son oracle et ses dieux, Paris, Les Belles Lettres, 1976, p. 211.

51 PIND., Ol., II, 42-44.

52 SoPH., O.R., 1529-1530. 
Sentence dont l'histoire de Crésus racontée par Hérodote est la parfaite illustration. Chez Eschyle et Euripide le mythe offrirait plutôt une «fatalité hypothétique», selon la formule d'Edmond Lévy 53 . Laïos pouvait écouter l'avertissement lancé par le dieu et faire en sorte de ne pas engendrer d'enfant. C'est parce qu'il n'a pas respecté l'avertissement qu'il a mis en marche le destin ${ }^{54}$. Si nous avions conservé l'intégrité des pièces que les Tragiques ont tirées des autres mythes et non quelques misérables fragments, nous trouverions probablement les mêmes leçons. À l'époque de l'apogée de Delphes, les mythes de la précaution inutile, me semble-t-il, constituent donc moins une illustration de la fatalité qu'une glorification d'Apollon.

Université Paul Valéry

Alain MOREAU

Montpellier III

B.P. 5043

F - 34032 MONTPELLIER Cédex

53 E. LEVY, La fatalité dans le théâtre d'Eschyle, in BAGB (1969), n 4, p. 412.

54 Esch., Sept, 742-749 (voir supra, p. 262); EUR., Phén., 17-20 (voir note 2). 